\section{EFSUMB Newsletter}

\author{
European Federation of Societies for Ultrasound in Medicine \\ and Biology
}

\title{
New EFSUMB Statement on Paediatric CEUS
}

As most of you will be aware, the FDA in the United States took the unprecedented step of approving an ultrasound contrast agent (Lumason ${ }^{\mathrm{TM}} /$ SonoVue $^{\mathrm{TM}}$ ) for use not only in the adult patient with a focal liver lesion but also in the paediatric population. Clinical trials for the adult patient were conducted in the United States but no such paediatric trial was performed, and in fact there has been no clinical trial anywhere in the world to address this issue. The decision of the FDA was based entirely on safety data provided by European centres, where CEUS in children has been performed for a number of years with success. Numerous European based studies have reported the usefulness of this technique in liver, and elsewhere particularly in the management of blunt abdominal trauma in the child. There is no justification to subject the child to repeat CT examinations when the CEUS examination is so accurate in isolated solid abdominal organ injury.
The granting of a licence for the use of an ultrasound contrast agent in the United States will alter medical practice worldwide - ultrasound practitioners will be less hesitant in using ultrasound contrast agents in the child. The safety profile is well established in adults and will be similar in the child.

With this development EFSUMB has commissioned a position statement on the use of CEUS in children, bringing together vast experience across Europe in aspects of CEUS application in children, culminating in a 10 page document summarizing this experience and advocating expanded uses of CEUS in children. This is ready and has been accepted for publication in the European Journal of Ultrasound; and is now available on-line.

The statement covers the use of CEUS in focal liver lesions, blunt abdominal trauma and voiding cystourography - the 3

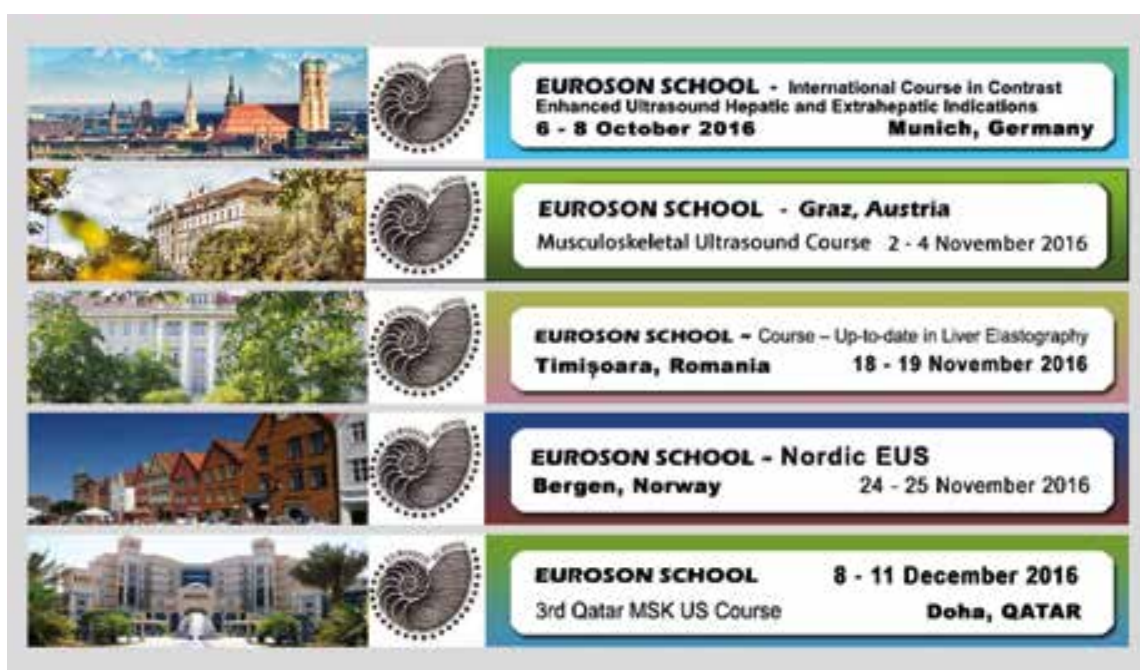

There is still time to apply to the following Schools with topics on CEUS, MSK, Elastography and EUS.
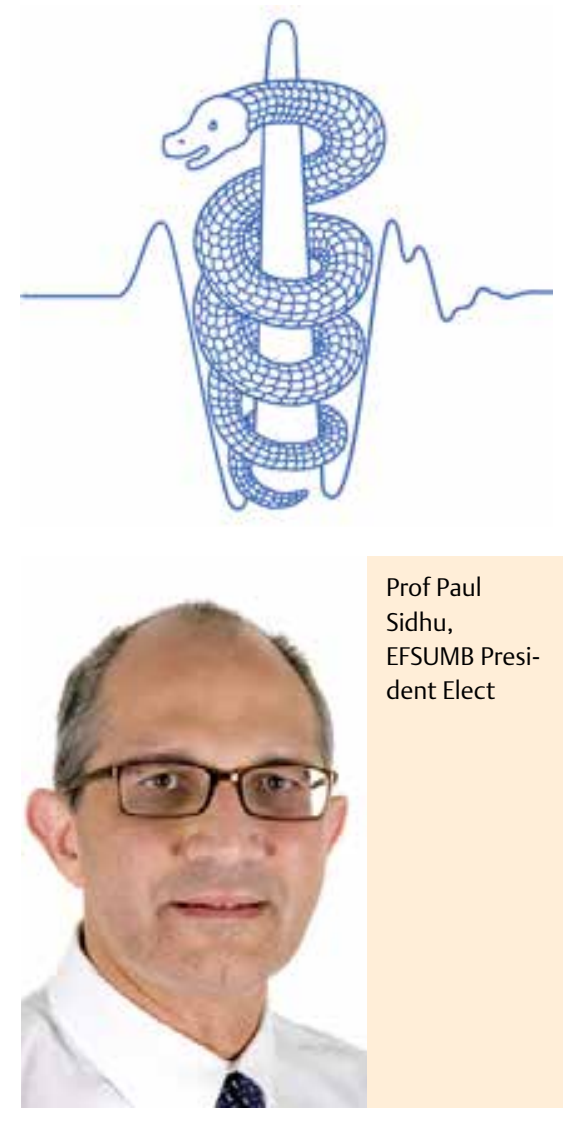

main areas of application currently. The statement speculates on the limited evidence in niche areas and predicts those applications that will increase and deliver useful clinical information. We expect this statement to benchmark the application of CEUS in children worldwide.

As ever, EFSUMB is grateful to the team of experts who committed time and energy to drafting this position statement, working rapidly and diligently over a short period of time. Congratulations to the whole of the EFSUMB ultrasound community who in many ways contributed to the expertise presented in the guidelines, by pushing innovation and exploring the application of ultrasound for the benefit and safety of patients.

\section{Prof Paul Sidhu \\ London, UK}

\section{EFSUMB}

Lynne Rudd

28 Portland Place, London

W1B 1LY, United Kingdom

Tel: +44 (0) 2070997140

Email: efsumb@efsumb.org 